\title{
Inequality and Welfare Evaluation of Heterogeneous Income Distributions
}

\author{
Anthony Shorrocks*
}

January 2004

\begin{abstract}
This paper establishes the principles which should govern the welfare and inequality analysis of heterogeneous income distributions. Two basic criteria-the 'equity preference' condition and the 'compensation principle' - are shown to be fundamentally incompatible. The paper favours the latter, thereby vindicating the traditional method of dealing with heterogeneous samples. However, inequality and welfare comparisons will usually be well defined only if equivalent incomes are obtained using constant scale factors; and researchers will need to distinguish clearly between inequality of nominal incomes and inequality of living standards. Furthermore, household observations must always be weighted according to family size.
\end{abstract}

Keywords: income distribution, inequality, living standards, needs JEL classification: I31, I32

Copyright (C) UNU-WIDER 2004

*UNU-WIDER, Helsinki.

This study has been prepared within the UNU-WIDER project on New Directions in Development Economics.

UNU-WIDER acknowledges the financial contributions to the research programme by the governments of Denmark (Royal Ministry of Foreign Affairs), Finland (Ministry for Foreign Affairs), Norway (Royal Ministry of Foreign Affairs), Sweden (Swedish International Development Cooperation Agency-Sida) and the United Kingdom (Department for International Development). 
The World Institute for Development Economics Research (WIDER) was established by the United Nations University (UNU) as its first research and training centre and started work in Helsinki, Finland in 1985. The Institute undertakes applied research and policy analysis on structural changes affecting the developing and transitional economies, provides a forum for the advocacy of policies leading to robust, equitable and environmentally sustainable growth, and promotes capacity strengthening and training in the field of economic and social policy-making. Work is carried out by staff researchers and visiting scholars in Helsinki and through networks of collaborating scholars and institutions around the world.

www.wider.unu.edu publications@wider.unu.edu

UNU World Institute for Development Economics Research (UNU-WIDER)

Katajanokanlaituri 6 B, 00160 Helsinki, Finland

Printed at UNU-WIDER, Helsinki

The views expressed in this publication are those of the author(s). Publication does not imply endorsement by the Institute or the United Nations University, nor by the programme/project sponsors, of any of the views expressed. 


\section{Introduction ${ }^{1}$}

A wide variety of tools for distributional analysis have developed from the original insights provided by Kolm (1969), Atkinson (1970) and Sen (1973). For inequality comparisons, Lorenz dominance is accepted as the criterion for 'unambiguous' or 'value-free' inequality rankings. When more detailed analysis is required, numerous 'Lorenz consistent' inequality indices available for application. In the context of welfare evaluation, a similar 'unambiguous' ranking criterion is provided by generalised Lorenz dominance (Shorrocks, 1983), and this can be supplemented when necessary with specific welfare indicators that respect the generalised Lorenz ordering.

While this array of tools may seem sufficient for everyday needs, they have been developed for a so-called homogeneous population whose members are assumed to be identical in all respects except for a uni-dimensional measure of income (or an alternative scalar measure of resources). This is not a comfortable restriction given the variety of personal, household and environmental factors, such as household size, leisure hours and local commodity prices, which applied economists often wish to take into account when making welfare judgements. To accommodate a heterogeneous population - i.e. one containing income units with diverse and welfare-relevant non-income characteristics — researchers typically adopt a two-stage procedure, first adjusting the raw income figures to compensate for differences in non-income factors, and then applying the standard tools to the set of 'equivalent incomes', treating the revised income values as if they had been obtained for a homogeneous population. The first stage of this procedure has been subject to a great deal of refinement, especially with regard to the scale factors used to adjust for household size. ${ }^{2}$ Until relatively recently, however, the second stage of the procedure received little attention, despite the absence of a clear theoretical foundation for

\footnotetext{
1 This is a revised and updated version of a paper which was written in 1995 and circulated as Discussion Paper No. 447 of the Department of Economics of Essex University. Thanks are due to the UK ESRC for financial support under contract H52427501994, and to James Foster, John Weymark, Paul Glewwe and two referees of this journal for helpful comments and suggestions.

2 See, for example, Whiteford (1985), Buhmann et al (1988), Conniffe (1992), Nelson (1993), Nicol (1994), and Pendakur (1999).
} 
the methods commonly employed in practice.

This paper attempts to provide a rigorous foundation for the inequality and welfare evaluation of income distributions in a heterogeneous environment. Given that members of the population are characterised by more than one welfare-relevant attribute, the topic might be viewed as a multi-dimensional problem of the type explored by Kolm (1977), Atkinson and Bourguignon (1982, 1987), Maasoumi (1986), and others. ${ }^{3}$ However, multi-dimensional analysis typically has a strong ordinal flavour, placing few constraints on the way in which personal and environmental attributes can be traded-off against each other. In contrast, use of equivalence scale factors and similar constructs to make compensatory income adjustments presupposes that such trade-offs are always possible. This is the starting point of the approach adopted in this paper

The need for a reappraisal of conventional methodology became more evident following Glewwe (1991) who showed how a regressive income transfer from a less well-off household can, in certain circumstances, reduce the measured level of inequality. Ebert (1995, 1997, 1999) pursues further the logical consequences of Glewwe's example, by constructing heterogeneous indices of welfare and inequality which respond in the expected way to regressive income transfers, and by identifying circumstances in which these indices agree on their ranking of any given pair of distributions. ${ }^{4}$ Similar criteria were also proposed by Pyatt (1990). However the procedures which they recommended are not those employed in practice by researchers undertaking distributional analysis.

The paper begins with a discussion of measures of living standards which allow members of the population to be completely ordered according to their level of material well-being. This is followed in Section 3 by a brief summary of some of the well-known results for homogeneous income distributions. Section 4 describes various principles for heterogeneous inequality and

\footnotetext{
3 Subsequent contributions include Bourguignon (1989), Sen (1992), Jenkins and Lambert (1993), Tsui (1995, 1999, 2002), Maasoumi (1999), Ok and Lambert (1999), Mukherjee (2001), Lambert and Ramos (2002) and Fleurbaey et al. (2003).

4 See Ebert and Moyes (2003), Trannoy (2003) and Ooghe (2003) for discussions of related issues.
} 
welfare analysis, focusing in particular on two plausible axioms: the equity preference condition which says that welfare rises (and inequality falls) when income is transferred to those worse-off; and the compensation principle which demands that welfare and inequality remain unchanged whenever a member of the population is replaced by another with the same standard of living, but possibly different personal characteristics. While both of these properties seem intuitively appealing, they turn out to be compatible only in exceptional circumstances. So one has to be discarded.

For welfare analysis, the compensation principle seems the more compelling requirement. By dispensing with equity preference, Section 5 is able to vindicate certain procedures applied to heterogeneous welfare comparisons in the past. It is noted, however, that these procedures may not be well-founded unless living standards are homothetic. It is also demonstrated that the compensation principle is compatible with a less demanding equity criterion inspired by Sen's (1973) Weak Equity Axiom. For inequality comparisons, equity preference appears the more natural point of departure, so the inequality analysis of Section 6 proceeds on this basis. The results enable the contributions of Glewwe (1990) and Ebert $(1995,1997,1999)$ to be understood and evaluated. But the discussion also reveals a number of problems which cast doubt on the wisdom of continuing further along these lines. Section 7 spells out the implications of the preceding results for populations of households distinguished by family size, and the paper ends with a summary of the main conclusions.

\section{Living Standards and Equivalent Incomes}

Consider a population of $n \geq 2$ individuals indexed by the set $N=\{1,2, \ldots, n\}$, and suppose that the inequality and welfare relevant characteristics of person $i \in N$ are captured by per-capita nominal household income $y_{i} \in \mathbb{R}_{++}=(0, \infty)$ and a non-income parameter $\theta_{i}$, referred to as the 'personal type' and treated as a scalar variable, although it could equally well be a vector. It should be emphasised at the outset that the decision to take individuals (rather than households) as the economic unit, and per-capita household income as the measure of economic resources, 
does not prejudice the results in any way. ${ }^{5}$ The principal reason for basing the analysis on individuals is that the size of the population is not affected by the various ways in which individuals may group themselves into households. Per-capita household income is chosen as the resource variable in preference to household income in order that the values of $y_{i}$ summed across individuals yield the aggregate income of the population.

Individuals are completely ordered by their living standard $v(y, \theta)$, which may be regarded as a cardinal an interpersonally comparable indirect utility function with prices suppressed or absorbed into $\theta$. For all $\theta, v(\cdot, \theta)$ is assumed to be increasing and differentiable. ${ }^{6}$ Furthermore, the units in which $v$ is measured may be calibrated so that

$$
v\left(y, \theta^{*}\right)=y, \quad \text { for all } y>0
$$

for some arbitrarily chosen personal reference type $\theta^{*}$. If $v(\cdot, \theta)=v\left(\cdot, \theta^{*}\right)$ for all $\theta$, then variations in $\theta$ have no impact on living standards, and the environment may be said to be effectively homogeneous. To avoid this degenerate case it is assumed that

$$
v(y, \theta) \neq y \quad \text { for some } \theta \text { and some } y>0 .
$$

Living standards may also be defined in terms of the dual function $c(u, \theta)$, which represents the cost of achieving the living standard level $u$ by a person of type $\theta$, and which satisfies

$$
c(v(y, \theta), \theta)=y \quad \text { for all } \theta \text { and all } y>0
$$

with $c\left(u, \theta^{*}\right)=u$ for all $u>0$ under the normalisation (1). For expositional purposes it is more convenient here to frame the analysis in terms of the living standard $v$, although studies of household equivalence scales and cost of living indices tend to use the cost function $c$.

\footnotetext{
5 Indeed, the chosen method of representation is sufficiently general to encompass intra-household variations in living standards of the kind discussed by Lazear and Michael (1986, 1988), Haddad and Kanbur (1990) and Thomas (1990), although this is not an issue explored in this paper.

6 The differentiability assumption imposed here and elsewhere may be replaced by continuity for most of the results. However, differentiability is a prerequisite in Proposition 1, so it is convenient to include it at the outset.
} 
The reason why living standards depend on non-income factors is often attributed to differences in 'needs'. Specifically, if

$$
v\left(y, \theta_{1}\right)>v\left(y, \theta_{2}\right) \text { for all } y>0,
$$

then a $\theta_{2}$-type person can be said to have uniformly greater needs than a $\theta_{1}$-type person. The discussion of differences in needs has tended to focus on the issue of household size, with a presumption that a larger family requires a higher income to achieve any given standard of living. However, identifying $\theta$ with household size introduces a number of complications. To begin with, a larger family with the same household income has both a lower standard of living and a greater number of representatives in the population of individuals. Secondly, a larger family is usually thought to be better-off than a smaller family with the same per capita household income. So the decision to use per capita household income as the resource variable, combined with the definition of greater needs given in (4), suggests that larger families are less needy. Interpretation of $\theta$ in terms of household size may therefore produce an element of confusion that is best avoided at this stage.

In the motivation for the Weak Equity Axiom, Sen (1973) uses the example of a physical handicap - specifically, the situation of a cripple - to convey the idea of greater need. By sidestepping the problems raised by having different sample weights attached to individual observations, this interpretation provides a clearer illustration of the central issue. More generally, (4) may be taken to mean that a $\theta_{2}$-type person is 'handicapped' or 'disadvantaged' relative to a $\theta_{1}$-type person, terminology which covers not only the situation of the cripple described by Sen, but also circumstances in which a $\theta_{2}$-type person may be said to face the handicap of higher prices, say, if this is the source of differences between $\theta_{1}$ and $\theta_{2}$.

Given an arbitrary reference type $\hat{\theta}$, the (living standard) equivalent income $e>0$ of a type $\theta$ person with income $y$ is given implicitly by

$$
v(e, \hat{\theta})=v(y, \theta)
$$

or explicitly by $e=c(v(y, \theta), \hat{\theta})$. It is assumed throughout that $\theta^{*}$ provides a valid reference type 
for equivalent income calculations, and the corresponding equivalent income values are indicated by an asterisk, so that

$$
y^{*}=c\left(v(y, \theta), \theta^{*}\right)=v(y, \theta)>0 \quad \text { for all } \theta \text { and all } y>0 \text {. }
$$

Conditions (1) and (6) imply that $y^{*}$ may attain any value in the interval $\mathbb{R}_{++}$. If equivalent incomes are also well defined for the reference type $\hat{\theta}$, then

$$
e=c(v(y, \theta), \hat{\theta})=c\left(y^{*}, \hat{\theta}\right)>0 \quad \text { for all } y^{*}>0 .
$$

Furthermore, from (5) it follows that $v(e, \hat{\theta})=v(y, \theta)=y^{*}$ must have a solution $e>0$ for any value $y^{*}>0$, and hence, using (6), that $v(y, \hat{\theta}) \rightarrow 0$ as $y \rightarrow 0$, and that $v(y, \hat{\theta}) \rightarrow \infty$ as $y \rightarrow \infty$. More generally, if equivalent incomes are well defined for any choice of reference type, as typically seems to be assumed, then

$$
\begin{aligned}
& \operatorname{Lim}_{y \rightarrow 0} v(y, \theta)=\operatorname{Lim}_{u \rightarrow 0} c(u, \theta)=0 \text { for all } \theta ; \\
& \operatorname{Lim}_{y \rightarrow \infty} v(y, \theta)=\operatorname{Lim}_{u \rightarrow \infty} c(u, \theta)=\infty \quad \text { for all } \theta .
\end{aligned}
$$

Although not strictly necessary, the boundary conditions (8) enable a number of the subsequent results to be stated more succinctly. Since nothing of substance is gained by omitting these restrictions, the remainder of the paper will focus on the set $V$ of living standard functions $v(y, \theta)$ which are increasing and differentiable in $y$, and which satisfy the normalisation condition (1), the heterogeneity requirement (2) and the boundary constraints (8).

Ideally, one would like to be able to offer procedures for inequality and welfare analysis of heterogeneous income distributions under a wide variety of specifications of living standards. This turns out to be an impossible goal. As will be demonstrated later, unless $v \in V$ is heavily circumscribed, distributional comparisons will either depend on the personal type used as the reference for the equivalent income calculations, or else require the selection of particular inequality or welfare indices, decisions not best left to the idiosyncratic choices of individual researchers. To avoid these problems it is usually necessary that living standards have the homothetic form 


$$
v(y, \theta)=y / a(\theta) ; c(u, \theta)=a(\theta) u, \quad a(\theta)>0 .
$$

Compliance with (9) implies that equivalent incomes based on $\theta^{*}$ (or, indeed, any other choice of reference type) are obtained by deflating reported incomes by a constant scale factor independent of the level of income.

Homotheticity is a undoubtedly a serious constraint to impose on living standards. However, its practical significance for distributional evaluation is mitigated by the fact that most empirical applications to heterogeneous populations adopt this restriction. In particular, adjustments to compensate for differences in household size invariably employ a constant household equivalence scale factor $A$, say, which, for households of size $m$ with household income $m y$, would yield $a(\theta)=A / m$ in (9). Similarly, if $\theta$ is designed to capture commodity price variations, then nominal incomes are typically deflated by a relative price index $P$ which does not vary with income. Following Lewbel (1989), equivalence scale factors that conform with (9) may be said to have an IB (independent of base level utility or income) form.

\section{The Evaluation of Homogeneous Income Distributions}

Before proceeding to a detailed analysis of heterogeneous populations it is necessary to review some of the tools which have been developed for homogeneous distributions and which have traditionally been applied also to heterogeneous populations once reported incomes are converted into their equivalent income values. Denoting by $\mathscr{F}$ the set of cumulative distribution functions on $\mathbb{R}_{++}$with finite mean, the Lorenz curve for $F \in \mathscr{F}$ is defined by

$$
L(F ; p)=\frac{1}{\mu(F)} \int_{0}^{p} F^{-1}(q) d q, \quad p \in[0,1]
$$

where $\mu(F)=\int_{0}^{1} F^{-1}(q) d q$ is the mean of $F$. The distribution $F$ is said to (weakly) Lorenz dominate $\tilde{F}$ (written $F \geq_{\mathrm{L}} \tilde{F}$ ) if and only if

$$
L(F ; p) \geq L(\tilde{F} ; p) \text { for all } p \in[0,1]
$$


In a similar fashion, the generalised Lorenz curve for $F \in \mathscr{F}$ is defined by

$$
G L(F ; p)=\int_{0}^{p} F^{-1}(q) d q=\mu(F) L(F ; p), \quad p \in[0,1]
$$

and $F$ is said to (weakly) generalised Lorenz dominate $\tilde{F}$ (written $F \geq_{\mathrm{GL}} \tilde{F}$ ) if and only if

$$
G L(F ; p) \geq G L(\tilde{F} ; p) \text { for all } p \in[0,1]
$$

For the distributions in $\mathscr{F}$, generalised Lorenz dominance is equivalent to second degree stochastic dominance; it is also equivalent to weak majorisation from below (see Marshall and Olkin, 1979) when the distributions correspond to equal weighted samples of income observations.

In practice, researchers are most likely to confront distributional data consisting of a set of income values $\mathbf{x}=\left(x_{1}, \ldots, x_{n}\right)$ together with the associated sample weights $\mathbf{w}=\left(w_{1}, \ldots, w_{n}\right)$. The corresponding cdf is then the step function given by

$$
F(t)=\sum_{i=1}^{n} w_{i} \bar{F}\left(t ; x_{i}\right) / \sum_{i=1}^{n} w_{i}, \quad t>0,
$$

where

$$
\bar{F}(t ; x)= \begin{cases}0 & t<x \\ 1 & t \geq x\end{cases}
$$

is the step function in $\mathscr{F}$ with a single jump at the point $x$. In these circumstances it is convenient to regard $(\mathbf{x} \mid \mathbf{w})$ as an alternative representation of the distribution $F$, so that $\mu(\mathbf{x} \mid \mathbf{w})$ may be used to denote the (weighted) mean income, and $(\mathbf{x} \mid \mathbf{w}) \geq_{\mathrm{L}}(\tilde{\mathbf{x}} \mid \tilde{\mathbf{w}})$ to indicate a Lorenz dominance relation between weighted samples. Denoting the dimension of the vector $\mathbf{x}$ by $n(\mathbf{x})$, and defining the sets of differentiable concave and convex functions

$$
\begin{gathered}
\Phi=\left\{\varphi: \mathbb{R}_{++} \rightarrow \mathbb{R} \mid \varphi^{\prime} \text { is positive and decreasing }\right\}, \\
\Psi=\left\{\psi: \mathbb{R}_{++} \rightarrow \mathbb{R} \mid \psi^{\prime} \text { is increasing }\right\},
\end{gathered}
$$

enables a central result in welfare and inequality analysis to be stated in a particularly simple manner. 
Lemma 1: Suppose $n(\mathbf{x})=n(\tilde{\mathbf{x}})=n$. Then

(a) $(\mathbf{x} \mid \mathbf{w}) \geq_{\mathrm{GL}}(\tilde{\mathbf{x}} \mid \tilde{\mathbf{w}})$ if and only if

$$
\sum_{i=1}^{n} w_{i} \varphi\left(x_{i}\right) / \sum_{i=1}^{n} w_{i} \geq \sum_{i=1}^{n} \tilde{w}_{i} \varphi\left(\tilde{x}_{i}\right) / \sum_{i=1}^{n} \tilde{w}_{i} \quad \text { for all } \varphi \in \Phi .
$$

(b) $(\mathbf{x} \mid \mathbf{w}) \geq_{\mathrm{L}}(\tilde{\mathbf{x}} \mid \tilde{\mathbf{w}})$ if and only if

$$
\sum_{i=1}^{n} w_{i} \psi\left(\frac{x_{i}}{\mu(\mathbf{x} \mid \mathbf{w})}\right) / \sum_{i=1}^{n} w_{i} \leq \sum_{i=1}^{n} \tilde{w}_{i} \psi\left(\frac{\tilde{x}_{i}}{\mu(\tilde{\mathbf{x}} \mid \tilde{\mathbf{w}})}\right) / \sum_{i=1}^{n} \tilde{w}_{i} \quad \text { for all } \psi \in \Psi .^{7}
$$

Most theoretical analysis of distributions is undertaken in an even simpler framework consisting of equally weighted samples of $n$ income observations, in which case w may be viewed as an $n$-dimensional vector of 1 's, denoted here by $\mathbf{1}$. This allows an $n$-person income distribution to be represented by $\mathbf{x} \in \mathbb{R}_{++}^{n}$, rather than $(\mathbf{x} \mid \mathbf{1})$, and Lorenz dominance and generalised Lorenz dominance to be expressed in the form $\mathbf{x} z_{\mathrm{L}} \tilde{\mathbf{x}}$ and $\mathbf{x} z_{\mathrm{GL}} \tilde{\mathbf{x}}$, respectively.

Welfare functions for equally weighted samples are usually assumed to belong to the set

$$
\mathscr{W}=\left\{W: \mathbb{R}_{++}^{n} \rightarrow \mathbb{R} \mid W \text { is increasing and strictly Schur concave }\right\}
$$

The requirement that $W \in \mathscr{W}$ is strictly Schur concave serves as a convenient shorthand for two important properties: $W$ is symmetric, so that $W(\mathbf{x})=W(\mathbf{x} \Pi)$ for all permutation matrices $\Pi$; and $W$ prefers a more equal distribution in the sense that $W(\mathbf{x})<W(\tilde{\mathbf{x}})$ whenever $\mathbf{x}$ is obtained from $\tilde{\mathbf{x}}$ by a (mean-preserving) regressive income transfer or, in other words, whenever $\mathbf{x}, \tilde{\mathbf{x}} \in \mathbb{R}_{++}^{n}$ satisfy

$$
\mu(\mathbf{x})=\mu(\tilde{\mathbf{x}}) ; \quad x_{i}<\tilde{x}_{i} \leq \tilde{x}_{j}<x_{j} ; \quad \text { and } x_{k}=\tilde{x}_{k} \text { for } k \neq i, j .
$$

Similarly, measures of inequality for distributions $\mathbf{x} \in \mathbb{R}_{++}^{n}$ are typically assumed to be drawn from the set

7 Lemma 1(a) is a restatement of a familiar result on second degree stochastic dominance: see, for example, Fishburn and Vickson (1978). Lemma 1(b) is a slight modification of Corollary 3.2.1 of Arnold (1987). 


$$
\mathscr{I}=\left\{I: \mathbb{R}_{++}^{n} \rightarrow \mathbb{R} \mid I \text { is homogeneous of degree zero and strictly Schur convex }\right\},^{8}
$$

where the requirement that $I$ is strictly Schur convex corresponds to the assumptions that $I$ is symmetric and that $I(\mathbf{x})>I(\tilde{\mathbf{x}})$ whenever $\mathbf{x}$ is obtained from $\tilde{\mathbf{x}}$ by a (mean-preserving) regressive income transfer. Members of both $\mathscr{W}$ and $\mathscr{I}$ therefore comply with the so-called Pigou-Dalton condition:

Pigou-Dalton Condition: The distribution $\mathbf{x}$ has lower welfare and higher inequality than $\tilde{\mathbf{x}}$ whenever $\mathbf{x}$ is obtained from $\tilde{\mathbf{x}}$ by a (mean-preserving) regressive income transfer.

A unanimous ranking by members of $\mathscr{W}$ (or $\mathscr{I}$ ) is taken to indicate an 'unambiguous' welfare (or inequality) ordering. As is well known, generalised Lorenz dominance and Lorenz dominance are alternative characterisations of these unanimity orderings, so that

Lemma 2: Suppose $\mathbf{x}, \tilde{\mathbf{x}} \in \mathbb{R}_{++}^{n}$. Then

$$
\begin{aligned}
& \mathbf{x} \geq_{\mathrm{GL}} \tilde{\mathbf{x}} \quad \text { if and only if } W(\mathbf{x}) \geq W(\tilde{\mathbf{x}}) \quad \text { for all } W \in \mathscr{W} ; \\
& \mathbf{x} \geq_{\mathrm{L}} \tilde{\mathbf{x}} \quad \text { if and only if } \quad I(\mathbf{x}) \leq I(\tilde{\mathbf{x}}) \quad \text { for all } I \in \mathscr{I} .{ }^{9}
\end{aligned}
$$

In the homogeneous framework it may also be noted that, for distributions with identical means, welfare and inequality are inversely related. For if $\mu(\mathbf{x})=\mu(\tilde{\mathbf{x}})$, then the definitions of Lorenz dominance and generalised Lorenz dominance given by (11) and (13) imply

$$
\mathbf{x} \geq_{\mathrm{GL}} \tilde{\mathbf{x}} \text { if and only if } \mathbf{x} \geq_{\mathrm{L}} \tilde{\mathbf{x}} \text {. }
$$

The principal aim of this paper is to extend the analysis outlined above to a heterogeneous environment.

\footnotetext{
${ }^{8}$ With a fixed sample size, it is not necessary to assume the replication invariance property commonly incorporated in $\mathscr{W}$ and $\mathscr{A}$.

${ }^{9}$ Condition (20a) is established in Shorrocks (1983), while (20b) appears in Foster (1985).
} 


\section{Criteria for Heterogeneous Comparisons}

For an $n$-person sample from a heterogeneous population, the distribution of attributes is denoted by the partitioned vector $(\mathbf{y} ; \boldsymbol{\theta})=\left(y_{1}, \ldots, y_{\mathrm{n}} ; \theta_{1}, \ldots, \theta_{\mathrm{n}}\right)$, and the associated welfare and inequality values are represented by expressions of the form $W(\mathbf{y} ; \boldsymbol{\theta})$ and $I(\mathbf{y} ; \boldsymbol{\theta})$, respectively. If $\theta_{i}=\theta_{j}$ for all $i, j \in N$, then $(\mathbf{y} ; \boldsymbol{\theta})$ is a homogeneous sample to which the analysis of Section 3 can be applied. In particular, it is assumed that

$$
\begin{gathered}
W(\because \theta \mathbf{1}) \in \mathscr{W} \quad \text { for all } \theta ; \text { and } \\
I(\cdot ; \theta \mathbf{1}) \in \mathscr{I} \quad \text { for all } \theta,
\end{gathered}
$$

where $\mathscr{W}$ and $\mathscr{I}$ are given by (17) and (19). In this respect, the properties of heterogeneous indices are extensions of those imposed in a homogeneous environment.

A number of elementary properties may be defined by saying that the sample $(\mathbf{y} ; \boldsymbol{\theta})$ is obtained from $(\tilde{\mathbf{y}} ; \tilde{\boldsymbol{\theta}})$ by a permutation if $(\mathbf{y} ; \boldsymbol{\theta})=(\tilde{\mathbf{y}} \Pi ; \tilde{\boldsymbol{\theta}} \Pi)$ for some permutation matrix $\Pi$; by an income increment if $y_{i} \geq \tilde{y}_{i}$ for all $i \in N$ and $y_{i}>\tilde{y}_{i}$ for some $i \in N$; and by income scaling if $(\mathbf{y} ; \boldsymbol{\theta})=(\lambda \tilde{\mathbf{y}} ; \tilde{\boldsymbol{\theta}})$ for some $\lambda>0$. Then a heterogeneous welfare index $W$ is symmetric if $W(\mathbf{y} ; \boldsymbol{\theta})$ $=W(\tilde{\mathbf{y}} ; \tilde{\boldsymbol{\theta}})$ whenever $(\mathbf{y} ; \boldsymbol{\theta})$ is obtained from $(\tilde{\mathbf{y}} ; \tilde{\boldsymbol{\theta}})$ by a permutation, and increasing in income if $W(\mathbf{y} ; \boldsymbol{\theta})>W(\tilde{\mathbf{y}} ; \tilde{\boldsymbol{\theta}})$ whenever $(\mathbf{y} ; \boldsymbol{\theta})$ is obtained from $(\tilde{\mathbf{y}} ; \tilde{\boldsymbol{\theta}})$ by an income increment. Similarly, a heterogeneous inequality index $I$ is symmetric if $I(\mathbf{y} ; \boldsymbol{\theta})=I(\tilde{\mathbf{y}} ; \tilde{\boldsymbol{\theta}})$ whenever $(\mathbf{y} ; \boldsymbol{\theta})$ is obtained from $(\tilde{\mathbf{y}} ; \tilde{\boldsymbol{\theta}})$ by a permutation, and scale invariant in income if $I(\mathbf{y} ; \boldsymbol{\theta})=I(\tilde{\mathbf{y}} ; \tilde{\boldsymbol{\theta}})$ whenever $(\mathbf{y} ; \boldsymbol{\theta})$ is obtained from $(\tilde{\mathbf{y}} ; \tilde{\boldsymbol{\theta}})$ by income scaling.

Another natural property for both inequality and welfare indices is an appropriate analogue of the Pigou-Dalton condition. This may be formulated by considering a mean-preserving transfer of income to a person with a higher standard of living, and saying that $(\mathbf{y} ; \boldsymbol{\theta})$ is obtained from $(\tilde{\mathbf{y}} ; \boldsymbol{\theta})$ by a regressive income transfer from person $i$ to person $j$ if

$$
\mu(\mathbf{y})=\mu(\tilde{\mathbf{y}}) ; \quad v\left(y_{i}, \theta_{i}\right)<v\left(\tilde{y}_{i}, \theta_{i}\right) \leq v\left(\tilde{y}_{j}, \theta_{j}\right)<v\left(y_{j}, \theta_{j}\right) ; \quad \text { and } y_{k}=\tilde{y}_{k} \text { for } k \neq i, j
$$

A suitable extension of the Pigou-Dalton condition to the heterogeneous environment is then 
given by

Equity Preference: The distribution ( $\mathbf{y} ; \boldsymbol{\theta})$ has lower welfare (and/or higher inequality) than $(\tilde{\mathbf{y}} ; \boldsymbol{\theta})$ whenever $(\mathbf{y} ; \boldsymbol{\theta})$ is obtained from $(\tilde{\mathbf{y}} ; \boldsymbol{\theta})$ by a regressive income transfer.

If the welfare function $W$ is equity preferring and has partial derivatives with respect to income denoted by $W_{i}(\mathbf{y} ; \boldsymbol{\theta})=\partial W(\mathbf{y} ; \boldsymbol{\theta}) / \partial y_{i}$ for $i \in N$, then, by considering an arbitrarily small positive income transfer $d t$ from person $i$ to person $j$, it follows that

$$
d W=W_{j}(\mathbf{y} ; \boldsymbol{\theta}) d t-W_{i}(\mathbf{y} ; \boldsymbol{\theta}) d t<0 \quad \text { whenever } \quad v\left(y_{i}, \theta_{i}\right)<v\left(y_{j}, \theta_{j}\right),
$$

and equity preference may be expressed in the more convenient form

$$
W_{i}(\mathbf{y} ; \boldsymbol{\theta})>W_{j}(\mathbf{y} ; \boldsymbol{\theta}) \quad \text { if and only if } \quad v\left(y_{i}, \theta_{i}\right)<v\left(y_{j}, \theta_{j}\right) .
$$

Similarly, a differentiable inequality index is equity-preferring if

$$
I_{i}(\mathbf{y} ; \boldsymbol{\theta})<I_{j}(\mathbf{y} ; \boldsymbol{\theta}) \quad \text { if and only if } \quad v\left(y_{i}, \theta_{i}\right)<v\left(y_{j}, \theta_{j}\right) .
$$

It may be noted that successive application of equity preference is able to rank only those distributions which have the same aggregate income and the same pattern $\boldsymbol{\theta}$ of personal characteristics. To relax the latter constraint requires a method of deciding when a change of personal circumstances leaves overall welfare unaltered. This is accomplished by the compensation principle, defined as follows:

Compensation Principle: The distribution $(\mathbf{y} ; \boldsymbol{\theta})$ has the same level of welfare (and/or inequality) as the distribution $(\tilde{\mathbf{y}} ; \tilde{\boldsymbol{\theta}})$ whenever $v\left(y_{i}, \theta_{i}\right)=v\left(\tilde{y}_{i}, \tilde{\theta}_{i}\right)$ for all $i \in N$.

The compensation principle provides a way of capturing the welfare implications of changing personal circumstances and reflects the standard welfarist presumption that aggregate social welfare depends only on the utility levels of the population. It implies, for example, that if a person becomes physically handicapped, but his income is raised to compensate exactly for the disability, then social welfare remains the same. Similarly, in the context of household size considered later in Section 7, aggregate welfare is unchanged if a couple's divorce is 
accompanied by a rise in the per-capita household incomes which maintains the living standard of each of those involved. As will become evident, it is the compensation principle which vindicates the standard method of dealing with heterogeneous populations.

Although equity preference and the compensation principle both appear to offer plausible criteria for ranking heterogeneous distributions, they are almost incompatible as the following result demonstrates.

Proposition 1: Suppose $v \in V$. Then a heterogeneous welfare function $W$ with non-zero, continuous partial derivatives cannot respect both equity preference and the compensation principle.

Proof: Proposition 1 is established by showing that $W$ respects equity preference and the compensation principle only if the environment is effectively homogeneous.

If $W$ satisfies the compensation principle then replacing each person with their equivalent reference type yields

$$
W(\mathbf{y} ; \boldsymbol{\theta})=W\left(v\left(y_{1}, \theta_{1}\right), \ldots, v\left(y_{n}, \theta_{n}\right) ; \theta^{*} \mathbf{1}\right)=W\left(\mathbf{y}^{*} ; \theta^{*} \mathbf{1}\right) \text { for all } \mathbf{y}>\mathbf{0},
$$

and hence, using (3),

$$
W_{i}(\mathbf{y} ; \boldsymbol{\theta}) c_{1}\left(y_{i}^{*}, \theta_{i}\right)=W_{i}\left(\mathbf{y}^{*} ; \theta^{*} \mathbf{1}\right),
$$

where $c_{1}(u, \theta)=\partial c(u, \theta) / \partial u$. The equity preference condition (24) yields

$$
W_{i}(\mathbf{y} ; \boldsymbol{\theta})>W_{j}(\mathbf{y} ; \boldsymbol{\theta}) \quad \text { if and only if } \quad v\left(y_{i}, \theta_{i}\right)<v\left(y_{j}, \theta_{j}\right)
$$

in the context of the heterogeneous sample $(\mathbf{y} ; \boldsymbol{\theta})$ and

$$
W_{i}\left(\mathbf{y}^{*} ; \theta^{*} \mathbf{1}\right)>W_{j}\left(\mathbf{y}^{*} ; \theta^{*} \mathbf{1}\right) \quad \text { if and only if } \quad v\left(y_{i}^{*}, \theta^{*}\right)<v\left(y_{j}^{*}, \theta^{*}\right)
$$

when applied to $\left(\mathbf{y}^{*} ; \theta^{*} \mathbf{1}\right){ }^{10}$ But since $v\left(y_{i}^{*}, \theta^{*}\right)=y_{i}^{*}=v\left(y_{i}, \theta_{i}\right)$ for all $i$, it follows from (27)-(29)

${ }^{10}$ Note that (29) follows directly from the Pigou-Dalton condition, given (22a). 
that for any $u>0$ we may choose $y_{i}^{*}=y_{j}^{*}=u$ to obtain

$$
c_{1}\left(u, \theta_{i}\right)=\frac{W_{i}\left(\mathbf{y}^{*} ; \theta^{*} \mathbf{1}\right)}{W_{i}(\mathbf{y} ; \boldsymbol{\theta})}=\frac{W_{j}\left(\mathbf{y}^{*} ; \theta^{*} \mathbf{1}\right)}{W_{j}(\mathbf{y} ; \boldsymbol{\theta})}=c_{1}\left(u, \theta_{j}\right) \quad \text { for all } \theta_{i} \text { and } \theta_{j},
$$

and hence $c(u, \theta)=c\left(u, \theta^{*}\right)+\beta(\theta)=u+\beta(\theta)$, for some function $\beta(\theta)$. The boundary condition (8) then yields $c(u, \theta)=u$ for all $u$ and all $\theta$, which contradicts the assumption that $v$ satisfies the heterogeneity condition (2).

Figure 1 here

The intuition behind Proposition 1 may be illustrated by considering a simple two person population with homothetic living standards given by $v\left(y_{1}, \theta_{1}\right)=y_{1}$ and $v\left(y_{2}, \theta_{2}\right)=y_{2} / \lambda, \lambda>1$. Distributions with equal living standards may then be represented, as in Figure 1, by points on the line $O E$ through the origin with slope $\lambda$. The equity preference condition (28) requires the marginal social rate of substitution $W_{1}(\mathbf{y} ; \boldsymbol{\theta}) / W_{2}(\mathbf{y} ; \boldsymbol{\theta})$ to be less than one below $O E$, while the homogeneous (ie Pigou-Dalton) version (29) of the equity preference condition combined with (27) demands that the marginal social rate of substitution is greater than $\lambda$ above $O E$, and less than $\lambda$ below. These constraints cannot be met simultaneously if the slope of the social indifference curve changes continuously; hence the conflict between equity preference and the compensation principle revealed in Proposition 1.

If $W$ is not required to be continuously differentiable, then it is possible to construct examples of welfare functions which satisfy both equity preference and the compensation principle, by allowing the social indifference curves to be kinked at the points corresponding to equal living standards (as depicted by the curve $\mathrm{AA}^{\prime}$ in Figure 1). For instance, when applied to the example illustrated in Figure 1, the two person welfare function

$$
W(\mathbf{y} ; \boldsymbol{\theta})=\alpha\left[v\left(y_{1}, \theta_{1}\right)+v\left(y_{2}, \theta_{2}\right)\right]+(1-\alpha) \min \left\{v\left(y_{1}, \theta_{1}\right), v\left(y_{2}, \theta_{2}\right)\right\}, \quad \alpha \in(0,1),
$$

satisfies both properties when $\alpha<1 / \lambda$. So equity preference and the compensation principle are not strictly contradictory in the context of welfare comparisons. For practical purposes, however, 
it seems inevitable that one of these axioms must be discarded. Furthermore, the same conclusion must also be drawn for inequality comparisons, since the analogue of Proposition 1 may be established by considering a heterogeneous inequality index $I(\mathbf{y} ; \boldsymbol{\theta})$, and replacing $W(\cdot)$ by $-I(\cdot)$ in the proof of Proposition 1.

If the equity preference condition is abandoned, as will be recommended for heterogeneous welfare comparisons, it is still possible — and indeed required by (22) - to retain its homogeneous counterpart, the Pigou-Dalton condition. It is also possible to conceive other, less demanding, equity criteria which are more in harmony with the compensation principle. An obvious candidate here is the Weak Equity Axiom proposed by Sen (1973, 1997, pp 16-23). Sen argues that if two people have the same income, and if one is uniformly needier than the other, then an income transfer to the more needy person raises the level of social welfare. This requirement may be amended slightly, without violating the spirit of Sen's argument, by supposing instead that an income transfer from the needier person reduces social welfare.

Weak Equity Axiom: Consider a heterogeneous sample $(\tilde{\mathbf{y}} ; \boldsymbol{\theta})$, and suppose that $\tilde{y}_{i}=\tilde{y}_{j}$ and that person $i$ is uniformly needier than person $j$. Then the distribution $(\mathbf{y} ; \boldsymbol{\theta})$ has lower welfare (and higher inequality) than $(\tilde{\mathbf{y}} ; \boldsymbol{\theta})$ whenever $(\mathbf{y} ; \boldsymbol{\theta})$ is obtained from $(\tilde{\mathbf{y}} ; \boldsymbol{\theta})$ by a regressive transfer from person $i$ to person $j$.

In comparison with equity preference, it is clear that the weak equity axiom is a less demanding condition, since the welfare reducing impact of a regressive transfer is required to hold only in particular circumstances.

For the example illustrated in Figure 1, person 2 is uniformly needier than person 1, and distributions with equal incomes lie on the line $O X$ with unit slope. The Weak Equity Axiom therefore implies that the marginal social rate of substitution $W_{1}(\mathbf{y} ; \boldsymbol{\theta}) / W_{2}(\mathbf{y} ; \boldsymbol{\theta})$ is less than one below $O X$, rather than below $O E$, as required by equity preference. Replacing equity preference with the Weak Equity Axiom eliminates the conflict evident in Proposition 1, and allows the construction of welfare functions with smooth indifference curves like that depicted by $B B^{\prime}$ in Figure 1. For instance, any differentiable welfare function which is the sum of an increasing and 
$\log$ concave transformation of equivalent incomes satisfies both the compensation principle and the Weak Equity Axiom (see Proposition 3 below).

\section{Heterogeneous Welfare Comparisons}

If higher social welfare is taken to mean a higher overall standard of living in the population, it is difficult to see how the compensation principle can be discarded. However, equity preference is less compelling for several reasons. For example, if resources can be transferred costlessly, then equity preference implies that social welfare is maximised when living standards are equal. ${ }^{11}$ So at the social optimum the living standards of those with serious physical handicaps set an upper bound on what the rest of the population can achieve - a Rawlsian prescription that will not appeal to most people.

The basic problem here is that equity preference provides a plausible principle of equity, but social welfare depends on both equity and efficiency considerations. Those most handicapped are likely to face not only a higher cost of achieving a given standard of living, but also a higher marginal cost of raising their living standard. In this respect, they are a less efficient vehicle for converting resources into units of living standards, and hence units of social welfare. Given two individuals with the same income, it may be reasonable to argue, along with Sen (1973), that aggregate welfare can be improved by an income transfer to the needier person: the gain from greater equity may outweigh any efficiency loss. However, as noted in Section 4, equity preference is considerably stronger than the weak equity axiom, and takes no account whatsoever of the efficiency aspects.

In this section, the equity preference condition is disregarded, and attention confined to the set of welfare functions - denoted here by $\mathscr{W}^{*}$ — which are symmetric and increasing in income, and which satisfy the compensation principle and the homogeneity constraint (22a). Choosing $\hat{\theta}$ as the reference type, and using the compensation principle to replace $\left(y_{i}, \theta_{i}\right)$ with

11 See Ebert (1997) for further discussion of this point. 
$\left(c\left(y_{i}^{*}, \hat{\theta}\right), \hat{\theta}\right)$, enables $W \in \mathscr{W}^{*}$ to be written

$$
\begin{aligned}
W(\mathbf{y} ; \boldsymbol{\theta}) & =W\left(c\left(y_{1}^{*}, \hat{\theta}\right), \ldots, c\left(y_{n}^{*}, \hat{\theta}\right) ; \hat{\theta} \mathbf{1}\right) \\
& =W\left(y_{1}^{*}, \ldots, y_{n}^{*} ; \theta^{*} \mathbf{1}\right) \quad \text { when } \hat{\theta}=\theta^{*}
\end{aligned}
$$

The techniques developed for homogeneous samples may now be absorbed, via the right hand side of (32), into the analysis of heterogeneous populations. In effect, this describes the traditional procedure for dealing with heterogeneous samples: convert incomes into their equivalent income values, and then treat those values as if they had been obtained for a homogeneous population.

By analogy with the homogeneous case, $(\mathbf{y} ; \boldsymbol{\theta})$ may be said to be unambiguously weakly preferred to $(\tilde{\mathbf{y}} ; \tilde{\boldsymbol{\theta}})$ on welfare grounds (denoted here by $\left.(\mathbf{y} ; \boldsymbol{\theta}) \geq_{\mathrm{W}}(\tilde{\mathbf{y}} ; \tilde{\boldsymbol{\theta}})\right)$ if

$$
W(\mathbf{y} ; \boldsymbol{\theta}) \geq W(\tilde{\mathbf{y}} ; \tilde{\boldsymbol{\theta}})) \quad \text { for all } W \in \mathscr{P}^{*} .
$$

The compensation principle implies that (33) is equivalent to the requirement

$$
W\left(\mathbf{y}^{*} ; \theta^{*} \mathbf{1}\right) \geq W\left(\tilde{\mathbf{y}}^{*} ; \theta^{*} \mathbf{1}\right) \text { for all } W\left(\because \theta^{*} \mathbf{1}\right) \in \mathscr{W},
$$

which in turn holds if and only if

$$
\left(y_{1}^{*}, \ldots, y_{n}^{*}\right) \geq_{\mathrm{GL}}\left(\tilde{y}_{1}^{*}, \ldots, \tilde{y}_{n}^{*}\right)
$$

by Lemma 2. For this reason, traditional methods assume that $(\mathbf{y} ; \boldsymbol{\theta})$ weakly welfare dominates $(\tilde{\mathbf{y}} ; \tilde{\boldsymbol{\theta}})$ if and only if $\mathbf{y}^{*} \geq_{\mathrm{GL}} \tilde{\mathbf{y}}^{*}$. Thus the notion of an unambiguous welfare ordering not only translates readily to the heterogeneous environment, but does so in a way that allows the dominance relation to be validated via the generalised Lorenz criterion.

There is, however, a problem with this argument. If $\hat{\theta}$ rather than $\theta^{*}$ is selected as the reference type, then statements (34) and (35) require $\theta^{*}$ to be replaced by $\hat{\theta}$, and the terms $y_{i}^{*}$ and $\tilde{y}_{i}^{*}$ by $T\left(y_{i}^{*}\right)$ and $T\left(\tilde{y}_{i}^{*}\right)$, respectively, where $T(u)=c(u, \hat{\theta})$. The 'unambiguous welfare ordering' $\geq_{\mathrm{W}}$ is therefore well defined for alternative choices of the reference type only if (35) is equivalent to 


$$
\left(T\left(y_{1}^{*}\right), \ldots, T\left(y_{n}^{*}\right)\right) \geq_{\mathrm{GL}}\left(T\left(\tilde{y}_{1}^{*}\right), \ldots, T\left(\tilde{y}_{n}^{*}\right)\right) .
$$

Expressed slightly differently, the coincidence between (35) and (36) requires second degree stochastic dominance to be preserved by the transformation $T$, and also by its inverse $T^{-1}$, which is true if and only if $T$ is increasing and affine (Moyes and Shorrocks (1994), Proposition 4). Hence

$$
T(u)=c(u, \hat{\theta})=a(\hat{\theta}) u+b(\hat{\theta}), \quad a(\hat{\theta})>0,
$$

which the boundary condition (8) reduces to the homothetic form $c(u, \hat{\theta})=a(\hat{\theta}) u$. Furthermore, as the equivalence between (35) and (36) follows trivially when $v$ satisfies (9), the converse result also holds. Hence

Proposition 2: Suppose $v \in V$. Then the unanimity welfare ordering $\geq_{\mathrm{W}}$ is well defined if and only if $v$ is homothetic. ${ }^{12}$

The lesson to be drawn from Proposition 2 is that generalised Lorenz dominance will not translate to the heterogeneous environment in a consistent manner unless living standards are given by (9). ${ }^{13,14}$ Those who have undertaken heterogeneous welfare comparisons in the past using IB scale factors may take comfort from the fact that their procedures have a sound theoretical foundation. But those who wish to accommodate more flexible representations of living standards clearly face a number of additional hurdles.

\footnotetext{
12 This result (and also Proposition 4 below) is not entirely unexpected, given the frequency with which homotheticity restrictions recur in discussions of interpersonal welfare comparisons: see, for instance, Roberts (1980), Lewbel (1991), Pollack (1991) and Blackorby and Donaldson (1993). The principal difference here is the concern with the (incomplete) unanimity ordering $\geq_{\mathrm{W}}$, rather than a complete ordering or a specific welfare function.

13 This contrasts with the claim by Glewwe (1991, p. 212) that 'it makes no difference which method is used to estimate the equivalence scales, or what functional form they take'.

14 The same issue may be viewed from a slightly different perspective by noting that the properties of the welfare function $W(\because \hat{\theta} \mathbf{1})$ defined for $\hat{\theta}$-type equivalent incomes are not automatically inherited by the corresponding welfare function $W\left(\bullet ; \theta^{*} \mathbf{1}\right)$ defined for $\theta^{*}$-type incomes. In particular, if $W(\bullet ; \hat{\theta} \mathbf{1})$ and $W\left(\bullet ; \theta^{*} \mathbf{1}\right)$ are related by $(32)$, then — temporarily disregarding condition $(22 \mathrm{a})$ — it is not necessarily true that $W\left(\cdot ; \theta^{*} \mathbf{1}\right) \in \mathscr{W}$ implies $W(\cdot ; \hat{\theta} \mathbf{1}) \in \mathscr{W}$ (and vice-versa) unless $v$ is homothetic.
} 
While equity preference is difficult to reconcile with the compensation principle, Section 4 noted that the Weak Equity Axiom is a less demanding equity criterion which is compatible with the compensation principle in certain circumstances. To identify examples of relevant circumstances, it is convenient to restrict attention to homothetic living standards, and to suppose that welfare functions have the additive form

$$
W(\mathbf{y} ; \boldsymbol{\theta})=\sum_{i \in N} u\left(y_{i} / a\left(\theta_{i}\right)\right), \quad u \in \Phi
$$

where $\Phi$ is the set of differentiable concave functions given by (16a). These welfare functions respect the compensation principle, and also satisfy requirement (22a). It is now possible to establish:

Proposition 3: Suppose $v \in V$ is homothetic, and let $W$ be given by (38). Then $W$ satisfies the Weak Equity Axiom if and only if $u$ is strictly log concave.

Proof: Set $a_{i}=a\left(\theta_{i}\right)$ for all i, and define $\varphi(s)=u\left(\mathrm{e}^{s}\right)=$ for $s \in \mathbb{R}$. Then (38) may be rewritten

$$
W(\mathbf{y} ; \boldsymbol{\theta})=\sum_{i \in N} \varphi\left(\ln y_{i}-\ln a_{i}\right) .
$$

Consider a heterogeneous sample $(\mathbf{y} ; \boldsymbol{\theta})$ for which $y_{i}=y_{j}=y$, say, and also $a_{i}>a_{j}$, so that person $i$ is uniformly needier than person $j$. By the Weak Equity Axiom, a regressive transfer of amount $t$ from person $i$ reduces welfare by the (negative) amount

$$
\Delta W=\varphi\left(\ln (y+t)-\ln a_{j}\right)+\varphi\left(\ln (y-t)-\ln a_{i}\right)-\varphi\left(\ln y-\ln a_{j}\right)-\varphi\left(\ln y-\ln a_{i}\right) .
$$

So the Weak Equity Axiom is satisfied if and only if

$$
\int_{0}^{t}\left[\frac{\varphi^{\prime}\left(\ln (y+s)-\ln a_{j}\right)}{y+s}-\frac{\varphi^{\prime}\left(\ln (y-s)-\ln a_{i}\right)}{y-s}\right] d s<0
$$

whenever $y>0, t \in(0, y)$, and $a_{i}>a_{j}$.

Now if $u$ is $\log$ concave, then $\varphi$ is concave, and hence $\varphi^{\prime}\left(\ln (y+s)-\ln a_{j}\right)<\varphi^{\prime}(\ln (y-s)-$ $\left.\ln a_{i}\right)$ whenever $y>0, s \in(0, y)$, and $a_{i}>a_{j}$. So (41) holds, and $W$ satisfies the Weak Equity 
Axiom. Conversely, by taking the limit as $t \rightarrow 0$, condition (41) implies

$$
\frac{\varphi^{\prime}\left(\ln y-\ln a_{j}\right)}{y}-\frac{\varphi^{\prime}\left(\ln y-\ln a_{i}\right)}{y}<0 \quad \text { whenever } y>0 \text { and } a_{i}>a_{j},
$$

from which it follows that $\varphi^{\prime}$ is decreasing. Hence $\varphi$ is strictly concave, and $u$ is strictly $\log$ concave. The proof of Proposition 3 is therefore complete. ${ }^{15}$

From Proposition 3 it follows that the welfare functions given by

$$
W(\mathbf{y} ; \boldsymbol{\theta})=\sum_{i \in N} \varphi\left(\ln y_{i}-\ln a\left(\theta_{i}\right)\right)=\sum_{i \in N} \varphi\left(\ln y_{i}^{*}\right), \quad \varphi \in \Phi,
$$

respect both the compensation principle and the Weak Equity Axiom when living standards are homothetic. Although these functions do not satisfy equity preference, equity considerations are still strongly represented, via the Pigou-Dalton condition for homogeneous samples and the Weak Equity Axiom for heterogeneous samples.

For homothetic living standards it has already been noted that $W(\mathbf{y} ; \boldsymbol{\theta}) \geq W(\tilde{\mathbf{y}} ; \tilde{\boldsymbol{\theta}}))$ for all $W \in \mathscr{W}^{*}$ if and only if $\mathbf{y}^{*} z_{\mathrm{GL}} \tilde{\mathbf{y}}^{*}$. Restricting attention to the subset of $\mathscr{W}^{*}$ which satisfy the Weak Equity Axiom yields a unanimity ordering which allows further pairs of heterogeneous samples to be conclusively ranked. While it is difficult to characterise this additional ranking power, a good indication is provided by the unanimity ordering derived from the log concave welfare functions given in (43). For this set of functions, $(\mathbf{y} ; \boldsymbol{\theta})$ is unambiguously weakly preferred to $(\tilde{\mathbf{y}} ; \tilde{\boldsymbol{\theta}})$ if and only if

$$
\sum_{i \in N} \varphi\left(\ln y_{i}^{*}\right) \geq \sum_{i \in N} \varphi\left(\ln \tilde{y}_{i}^{*}\right) \quad \text { for all } \varphi \in \Phi,
$$

which by Lemma 1(a) is equivalent to the requirement that

$$
\left(\ln y_{1}^{*}, \ldots, \ln y_{n}^{*}\right) \geq_{\mathrm{GL}}\left(\ln \tilde{y}_{1}^{*}, \ldots, \ln \tilde{y}_{n}^{*}\right) .
$$

Thus, for instance, it follows from Proposition 3 that:

\footnotetext{
15 Note that if $u$ is increasing and strictly $\log$ concave, then $u$ is also strictly concave, and hence a member of $\Phi$.
} 
Corollary: Suppose $v \in V$ is homothetic. Then $W(\mathbf{y} ; \boldsymbol{\theta}) \leq W(\tilde{\mathbf{y}} ; \tilde{\boldsymbol{\theta}})$ for all $W \in \mathscr{W}^{*}$ which satisfy the Weak Equity Axiom only if

$$
\left(\ln y_{1}-\ln a\left(\theta_{1}\right), \ldots, \ln y_{n}-\ln a\left(\theta_{n}\right)\right) \geq_{\mathrm{GL}}\left(\ln \tilde{y}_{1}-\ln a\left(\tilde{\theta}_{1}\right), \ldots, \ln \tilde{y}_{n}-\ln a\left(\tilde{\theta}_{n}\right)\right)
$$

\section{Heterogeneous Inequality Comparisons}

As noted earlier, equity preference and the compensation principle are not easily reconciled in the context of inequality analysis. However, the objections levelled against equity preference in the previous section have less force when inequality comparisons are considered. Indeed, if the focus of attention is inequality in the distribution of nominal incomes, it is difficult to fault the argument that measured inequality increases when a nominal income transfer is made to those better-off, as equity preference demands. In contrast, there seems less reason to accept the compensation principle as a fundamental requirement unless the concern is with inequality in living standards (or 'real incomes'), in which case it again becomes a plausible, and perhaps compelling, axiom. This point will be revisited later.

To examine the implications of equity preference, consider the set $\mathscr{I}^{*}$ of heterogeneous inequality indices which are symmetric, equity preferring, and scale invariant in incomes. As the members of $\mathscr{I}^{*}$ are extensions of homogeneous indices via (22b), this set of indices are unanimous in their ranking of a pair of homogeneous samples if and only if the Lorenz curves do not intersect (see Lemma 2). The unanimity ordering given by

$$
(\mathbf{y} ; \boldsymbol{\theta}) \geq_{\mathrm{I}}(\tilde{\mathbf{y}} ; \tilde{\boldsymbol{\theta}}) \quad \text { if and only if } \quad I(\mathbf{y} ; \boldsymbol{\theta}) \leq I(\tilde{\mathbf{y}} ; \tilde{\boldsymbol{\theta}}) \text { for all } I \in \mathscr{I}^{*}
$$

is therefore the natural counterpart to Lorenz dominance, and may be interpreted as indicating an unambiguous inequality ranking of heterogeneous samples. Since the definition of $\geq_{\text {I }}$ given in (47) does not immediately yield a feasible method of confirming whether inequality comparisons are unambiguous, it is necessary to explore alternative ways of obtaining a practical validation procedure. 
As in the previous section, problems arise when living standards are not homothetic. Here, however, the reasons do not concern alternative reference types for equivalent income calculations, but relate instead to the conjunction of equity preference and scale invariance. This may be seen by taking any $I \in \mathscr{I}^{*}$ and, for simplicity, assuming $I$ to be differentiable. From the equity preference condition (25) it then follows that

$$
\begin{gathered}
I_{i}(\mathbf{y} ; \boldsymbol{\theta})<I_{j}(\mathbf{y} ; \boldsymbol{\theta}) \quad \text { if and only if } \quad v\left(y_{i}, \theta_{i}\right)<v\left(y_{j}, \theta_{j}\right), \\
I_{i}(\lambda \mathbf{y} ; \boldsymbol{\theta})<I_{j}(\lambda \mathbf{y} ; \boldsymbol{\theta}) \quad \text { if and only if } \quad v\left(\lambda y_{i}, \theta_{i}\right)<v\left(\lambda y_{j}, \theta_{j}\right) .
\end{gathered}
$$

But scale invariance implies $I(\lambda \mathbf{y} ; \boldsymbol{\theta})=I(\mathbf{y} ; \boldsymbol{\theta})$, and hence $\lambda I_{i}(\lambda \mathbf{y} ; \boldsymbol{\theta})=I_{i}(\mathbf{y} ; \boldsymbol{\theta})$, which together with (48) yields

$$
v\left(\lambda y_{i}, \theta_{i}\right)<v\left(\lambda y_{j}, \theta_{j}\right) \quad \text { if and only if } \quad v\left(y_{i}, \theta_{i}\right)<v\left(y_{j}, \theta_{j}\right)
$$

So

$$
v(\lambda t, \theta)=f(v(t, \theta), \lambda)
$$

for some function $f(v, \lambda)$ increasing in $v$, from which it follows that $f(t, \lambda)=f\left(v\left(t, \theta^{*}\right), \lambda\right)=$ $v\left(\lambda t, \theta^{*}\right)=\lambda t$. Setting $a(\theta)=1 / v(1, \theta)$ then yields the homothetic form

$$
v(y, \theta)=f(v(1, \theta), y)=v(1, \theta) y=y / a(\theta) .
$$

given by (9). Thus

Proposition 4: Suppose $v \in V$. Then a differentiable heterogeneous inequality index $I$ is equity preferring and scale invariant in income only if $v$ is homothetic.

In view of Proposition 4, the remainder of this section assumes that living standards comply with the homotheticity (9) which, by suitably re-calibrating $\theta$, can be expressed more simply as

$$
v(y, \theta)=y / \theta=y^{*}, \quad \text { for all } \theta>0 \text { and } y>0 .
$$

with $\theta$ now representing a cardinal measure of 'need'. 
To attempt to characterise the unambiguous inequality ordering $\geq_{\mathrm{I}}$ defined in (47), consider the subset $\mathscr{G} \subset \mathscr{I}^{*}$ of indices which have the additive form

$$
I(\mathbf{y} ; \boldsymbol{\theta})=\sum_{i=1}^{n} \varphi\left(y_{i} / \mu(\mathbf{y}), \theta_{i}\right)
$$

where the partial derivative $\varphi_{1}(t, \theta)=\partial \varphi(t, \theta) / \partial y$ is increasing in $t$ for all $\theta$. The equity preference condition (48a) then yields

$$
\varphi_{1}\left(y_{i} / \mu(\mathbf{y}), \theta_{i}\right)<\varphi_{1}\left(y_{j} / \mu(\mathbf{y}), \theta_{j}\right) \quad \text { if and only if } \quad y_{i} / \theta_{i}<y_{j} / \theta_{j} \text {, }
$$

and hence

$$
\varphi_{1}(t, \theta)=\psi^{\prime}(t / \theta)
$$

for some increasing function $\psi^{\prime}$, which upon integration becomes

$$
\varphi(t, \theta)=\theta \psi(t / \theta)+\gamma(\theta)
$$

where $\psi$ is convex, and $\gamma$ is an arbitrary function. The subset $\mathscr{Z}$ may therefore be represented by

$$
I(\mathbf{y} ; \boldsymbol{\theta})=\sum_{i=1}^{n}\left\{\theta_{i} \psi\left(\frac{y_{i}}{\mu(y) \theta_{i}}\right)+\gamma\left(\theta_{i}\right)\right\}, \quad \psi \in \Psi
$$

where $\Psi$ is the set of differentiable convex functions defined by (16b). Recalling the notation for a weighted sample of $n$ observations introduced in Section 3, it is now possible to establish:

Proposition 5: Suppose that $v \in V$ has the homothetic form (52) and that $\boldsymbol{\theta}=\tilde{\boldsymbol{\theta}}$ is fixed. Then $(\mathbf{y} ; \boldsymbol{\theta}) \geq_{\mathrm{I}}(\tilde{\mathbf{y}} ; \tilde{\boldsymbol{\theta}})$ only if $\left(\mathbf{y}^{*} \mid \boldsymbol{\theta}\right) \geq_{\mathrm{L}}\left(\tilde{\mathbf{y}}^{*} \mid \tilde{\boldsymbol{\theta}}\right)$.

Proof: Assume that $(\mathbf{y} ; \boldsymbol{\theta}) \geq_{\mathrm{I}}(\tilde{\mathbf{y}} ; \boldsymbol{\theta})$ and consider any $\psi \in \Psi$. Define $\varphi(u)=\psi(u / \mu(\boldsymbol{\theta}))$ and note that $\psi \in \Psi$ implies $\varphi \in \Psi$. Since $\mathscr{\mathscr { J }}$ is a subset of $\mathscr{I}^{*}$, it follows from (47) and (57) that

$$
\sum_{i=1}^{n} \theta_{i} \varphi\left(\frac{y_{i}}{\mu(\mathbf{y}) \theta_{i}}\right) \leq \sum_{i=1}^{n} \theta_{i} \varphi\left(\frac{\tilde{y}_{i}}{\mu(\tilde{\mathbf{y}}) \theta_{i}}\right)
$$

Using the fact that $\mu(\mathbf{y})=\sum_{i} y_{i}^{*} \theta_{i} / n=\mu\left(\mathbf{y}^{*} \mid \boldsymbol{\theta}\right) \mu(\boldsymbol{\theta})$ yields 


$$
\sum_{i=1}^{n} \theta_{i} \psi\left(\frac{y_{i}^{*}}{\mu\left(\mathbf{y}^{*} \mid \boldsymbol{\theta}\right)}\right) \leq \sum_{i=1}^{n} \theta_{i} \psi\left(\frac{\tilde{y}_{i}^{*}}{\mu\left(\tilde{\mathbf{y}}^{*} \mid \boldsymbol{\theta}\right)}\right) \quad \text { for all } \psi \in \Psi
$$

and the result follows immediately by Lemma 1(b).

This result appears remarkable at first sight. For it suggests — at least when living standards satisfy condition (52) - that unambiguous inequality comparisons may be validated by treating the (equally weighted) heterogeneous sample as if it comprised a homogeneous set of equivalent income values with sample weights given by the scale factors. ${ }^{16}$ The intuition behind this result is not easy to fathom, and it may well be the case that no simple explanation is available.

Proposition 5 provides a valuable insight into the paradox discussed by Glewwe (1991). Consider the following pair of 3-person distributions:

\begin{tabular}{c|ccc|ccc}
$i$ & $y_{i}$ & $\theta_{i}$ & $y_{i}^{*}$ & $\tilde{y}_{i}$ & $\theta_{i}$ & $\tilde{y}_{i}^{*}$ \\
\hline 1 & 4 & 2 & 2 & 2 & 2 & 1 \\
2 & 2 & 1 & 2 & 4 & 1 & 4 \\
3 & 4 & 1 & 4 & 4 & 1 & 4 \\
\hline
\end{tabular}

In this example, somewhat simpler than that offered by Glewwe, $(\tilde{\mathbf{y}} ; \boldsymbol{\theta})$ is obtained from $(\mathbf{y} ; \boldsymbol{\theta})$ by a regressive transfer from person 1 to person 2 . So $(\tilde{\mathbf{y}} ; \boldsymbol{\theta})$ is more unequal than $(\mathbf{y} ; \boldsymbol{\theta})$ by equity preference. But the traditional method of deciding inequality rankings on the basis of the (unweighted) equivalent income values would infer that $\mathbf{y}^{*}$ does not Lorenz dominate $\tilde{\mathbf{y}}^{*}$ because the share of the bottom two-thirds of the sample is 4/8 compared to 5/9. Consequently, some (but not all) of the indices in the set $\mathscr{I}$ must attach a lower inequality value to $\tilde{\mathbf{y}}^{*}$ than to $\mathbf{y}^{*}$. In other words, a regressive transfer appears to lead to a reduction in inequality according to some perfectly acceptable measures.

The flaw in this argument is that traditional practice implicitly invokes the compensation

\footnotetext{
16 The observation that sample values have to be weighted by the scale factors in order to satisfy equity preference was first made by Pyatt (1990) in the context of welfare comparisons.
} 
principle. So the Glewwe paradox illustrates the fact that once equity preference is accepted, the compensation principle — and hence the traditional procedure for inequality comparisons must be abandoned. Proposition 5 suggests instead a comparison between the 'needs weighted' samples of equivalent incomes, and it may be confirmed that $\left(\mathbf{y}^{*} \mid \boldsymbol{\theta}\right)$ does indeed Lorenz dominate $\left(\tilde{\mathbf{y}}^{*} \mid \boldsymbol{\theta}\right)$ in the above example.

For this example it is also true that $(\mathbf{y} ; \boldsymbol{\theta}) \geq_{\mathrm{I}}(\tilde{\mathbf{y}} ; \boldsymbol{\theta})$ in the terminology of (47). However, Proposition 5 does not claim that $\left(\mathbf{y}^{*} \mid \boldsymbol{\theta}\right) \geq_{\mathrm{L}}\left(\tilde{\mathbf{y}}^{*} \mid \boldsymbol{\theta}\right)$ implies $(\mathbf{y} ; \boldsymbol{\theta}) \geq_{\mathrm{I}}(\tilde{\mathbf{y}} ; \boldsymbol{\theta})$; in fact the converse to Proposition 5 is not valid. For instance, if $\hat{\boldsymbol{\theta}}=(1,1,2), \mathbf{y}=(1,1,4)$ and $\tilde{\mathbf{y}}=(2,2,2)$, then $\left(\mathbf{y}^{*} \mid \hat{\boldsymbol{\theta}}\right)$ and $\left(\tilde{\mathbf{y}}^{*} \mid \hat{\boldsymbol{\theta}}\right)$ have identical Lorenz curves, and hence $\left(\mathbf{y}^{*} \mid \hat{\boldsymbol{\theta}}\right) z_{\mathrm{L}}\left(\tilde{\mathbf{y}}^{*} \mid \hat{\boldsymbol{\theta}}\right)$. But for ordered distributions $\mathbf{y} \in \mathbb{R}_{++}^{3}$ which satisfy $y_{1} \leq y_{2} \leq y_{3}$, the index

$$
I(\mathbf{y} ; \boldsymbol{\theta})=\sum_{i \in N} \sum_{j \in N} \theta_{i} \theta_{j} \max \left\{\frac{y_{i}}{\theta_{i} \mu^{*}}, \frac{y_{j}}{\theta_{j} \mu^{*}}\right\}+\theta_{3}{ }^{2} \max \left\{\frac{y_{3}}{\theta_{3} \mu^{*}}, 1\right\},
$$

with $\mu^{*}=\mu(\mathbf{y}) / \mu(\boldsymbol{\theta})$, satisfies the requirements for membership of $\mathscr{I}^{*}$ when $\boldsymbol{\theta}=\hat{\boldsymbol{\theta}},{ }^{17}$ and yields $I(\mathbf{y} ; \hat{\boldsymbol{\theta}})=72 / 3>64 / 3=I(\tilde{\mathbf{y}} ; \hat{\boldsymbol{\theta}})$ for the two samples defined above. So it is not true that $I(\mathbf{y} ; \hat{\boldsymbol{\theta}}) \leq$ $I(\tilde{\mathbf{y}} ; \hat{\boldsymbol{\theta}})$ for all $I \in \mathscr{I}^{*}$, and hence not true that $(\mathbf{y} ; \hat{\boldsymbol{\theta}}) \geq_{\mathrm{I}}(\tilde{\mathbf{y}} ; \hat{\boldsymbol{\theta}})$.

Another point to note is that Proposition 5 may be invoked only when the two samples have the same distribution of non-income attributes. The prerequisite in Proposition 5 may be relaxed slightly to allow $\boldsymbol{\theta}$ to be a permutation of $\tilde{\boldsymbol{\theta}}$, but this is still far too restrictive for practical purposes. To omit the requirement that $\boldsymbol{\theta}=\tilde{\boldsymbol{\theta}}$ in Proposition 5 - and hence permit comparisons between samples with different patterns of non-income attributes — it is necessary, either implicitly or explicitly, to assume that

$$
(\mathbf{y} ; \boldsymbol{\theta})=_{\mathrm{I}}(\tilde{\mathbf{y}} ; \tilde{\boldsymbol{\theta}}) \quad \text { if } \quad\left(\mathbf{y}^{*} \mid \boldsymbol{\theta}\right)=_{\mathrm{L}}\left(\tilde{\mathbf{y}}^{*} \mid \tilde{\boldsymbol{\theta}}\right),
$$

where $=_{I}$ and $=_{L}$ are the symmetric parts of $z_{I}$ and $z_{L}$, respectively. While (61) might be a reasonable requirement, it is not easy to see how it could be justified.

\footnotetext{
${ }^{17}$ Equity preference may be confirmed by considering the three separate cases $y_{1}{ }^{*} \leq y_{2}^{*} \leq y_{3}^{*} ; y_{1}^{*} \leq y_{3}^{*} \leq y_{2}^{*}$; and $y_{3}{ }^{*} \leq y_{1}{ }^{*} \leq y_{2}{ }^{*}$. For example, when $y_{1}{ }^{*} \leq y_{3}{ }^{*} \leq y_{2}{ }^{*}$, we have $\mu^{*} I=y_{1}+4 y_{3}+7 y_{2}+\max \left\{2 y_{3}, y_{1}+y_{2}+y_{3}\right\}$, from which it follows that $\mu^{*}\left(I_{i}(\mathbf{y} ; \tilde{\boldsymbol{\theta}})-I_{j}(\mathbf{y} ; \tilde{\boldsymbol{\theta}})\right)<0$ whenever $y_{i}^{*}<y_{j}^{*}$, as required by $(25)$.
} 
Ignore this hurdle for the moment, and suppose that 'needs-weighted Lorenz dominance' is adopted as the unambiguous inequality criterion for heterogeneous samples: in other words that $z_{I}$ is reformulated so that

$$
(\mathbf{y} ; \boldsymbol{\theta}) \geq_{\mathrm{I}}(\tilde{\mathbf{y}} ; \tilde{\boldsymbol{\theta}}) \quad \text { if and only if } \quad\left(\mathbf{y}^{*} \mid \boldsymbol{\theta}\right) \geq_{\mathrm{L}}\left(\tilde{\mathbf{y}}^{*} \mid \tilde{\boldsymbol{\theta}}\right)
$$

One consequence of adopting the compensation principle for welfare comparisons and equity preference for inequality comparisons is that, for samples with the same mean income, the traditional link between improvements in welfare and reductions in inequality no longer holds in a heterogeneous environment. Specifically, it is not the case that if $\mu(\mathbf{y})=\mu(\tilde{\mathbf{y}})$ then

$$
(\mathbf{y} ; \boldsymbol{\theta}) \geq_{\mathrm{W}}(\tilde{\mathbf{y}} ; \tilde{\boldsymbol{\theta}}) \quad \text { if and only if }(\tilde{\mathbf{y}} ; \tilde{\boldsymbol{\theta}}) \geq_{\mathrm{I}}(\mathbf{y} ; \boldsymbol{\theta})
$$

as might be suggested by (21). In fact, by considering the pair of samples given by

\begin{tabular}{c|ccc|ccc}
$i$ & $y_{i}$ & $\theta_{i}$ & $y_{i}^{*}$ & $\tilde{y}_{i}$ & $\tilde{\theta}_{i}$ & $\tilde{y}_{i}^{*}$ \\
\hline 1 & 2 & 2 & 1 & 1 & 1 & 1 \\
2 & 3 & 1 & 3 & 4 & 2 & 2 \\
\hline
\end{tabular}

it may be confirmed that $\mathbf{y}^{*} \geq_{\mathrm{GL}} \tilde{\mathbf{y}}^{*}$ and $\left(\tilde{\mathbf{y}}^{*} \mid \tilde{\boldsymbol{\theta}}\right) \geq_{\mathrm{L}}\left(\mathbf{y}^{*} \mid \boldsymbol{\theta}\right)$. Thus it is possible for one heterogeneous income distribution to exhibit both unambiguously higher welfare and unambiguously higher inequality than another income distribution with the same mean income. Clearly, this is a conclusion which will not be digested easily by those used to working in the homogeneous framework.

Another point to note is that if the only source of heterogeneity is the commodity prices which people face, then the criterion provided by (62) differs from that usually applied. In this example, assuming preferences are homothetic, $\theta_{i}$ may be taken to be the price index for person $i$, and these price indices will appear as sample weights in the Lorenz comparison between $\left(\mathbf{y}^{*} \mid \boldsymbol{\theta}\right)$ and $\left(\tilde{\mathbf{y}}^{*} \mid \tilde{\boldsymbol{\theta}}\right)$. This contrasts with the traditional procedure of simply comparing the (unweighted) samples of real incomes. To justify standard practice, it can be argued — quite reasonably — that 
our concern is with inequality of real incomes rather than nominal incomes, and that this can be accommodated by a minor change to the statement of equity preference, replacing "nominal income transfer' by 'real income transfer'.

Proceeding one step further, it may also be argued that our ultimate concern is with inequality of living standards, rather than inequality of incomes, either real or nominal. The compensation principle can then be resurrected, along with the link (63) between welfare and inequality, thereby justifying all the procedures traditionally employed. Given the problems encountered in attempting to extend Proposition 5, this solution may well be the preferred option. The main criticism is likely to concern the consequent reformulation of equity preference, which now involves transfers of units of living standard between households, an exercise not easy to envisage in practice (Coulter et al (1992), pp. 116-119). However, it should be noted that this version of equity preference follows directly from two more acceptable and comprehensible properties: the compensation principle and the Pigou-Dalton condition for homogeneous samples. As a consequence it does not need to be independently justified, and may be dropped altogether as a separate requirement.

\section{Differences in Family Size}

For reasons explained in Section 2, the interpretation of $\theta$ as family size has been eschewed up to now. But since family size is one of the principal sources of sample heterogeneity in welfare and inequality comparisons, it is worth exploring the implications of the preceding analysis for this important application. ${ }^{18}$

In order to do so, suppose that the set $N$ of individuals is partitioned into households, and let

\footnotetext{
${ }^{18}$ One referee has argued that the earlier results are not applicable to variations in household composition because members of the same family must have the same per capita household income, a constraint not recognised in the formulation of the problem. While the assumption of equal living standards within families places restrictions on the set of feasible patterns of heterogeneous income distributions, application of the earlier results is not rendered invalid simply because they can be applied in more general contexts.
} 
$N_{h}$ be the set of individuals in household $h$. The number of persons in household $h$ is denoted by $m_{h}$, and their aggregate income by $Y_{h}$. For simplicity, it is assumed that all members of household $h$ have the same living standard; that household income and family size are the only welfarerelevant characteristics; and that living standards are homothetic. Employing the notation of earlier sections then yields

$$
\begin{aligned}
y_{i}^{*} & =v\left(y_{i}, \theta_{i}\right)=v\left(Y_{h} / m_{h}, m_{h}\right) \quad \text { for } i \in N_{h} \\
& =\frac{Y_{h}}{m_{h} a\left(m_{h}\right)}=\frac{Y_{h}}{A\left(m_{h}\right)}=Y_{h}^{*},
\end{aligned}
$$

where $A(m)=a(m) m$ is the household equivalence scale factor traditionally used to deflate the (aggregate) income of a household with $m$ members.

The welfare analysis of Section 5 suggested that the heterogeneous sample $(\mathbf{y} ; \boldsymbol{\theta})$ is no worse than $(\tilde{\mathbf{y}} ; \tilde{\boldsymbol{\theta}}))$ if and only if $\mathbf{y}^{*} \geq_{\mathrm{GL}} \tilde{\mathbf{y}}^{*}$ which, by Lemma 1 , is equivalent to the condition

$$
\frac{1}{n} \sum_{i \in N} \varphi\left(y_{i}^{*}\right) \geq \frac{1}{n} \sum_{i \in N} \varphi\left(\tilde{y}_{i}^{*}\right) .
$$

Reformulating (65) in terms of households rather than individuals then yields

$$
\begin{gathered}
\frac{1}{n} \sum_{i \in N} \varphi\left(y_{i}^{*}\right)=\frac{1}{n} \sum_{h} \sum_{i \in N_{h}} \varphi\left(y_{i}^{*}\right) \\
=\sum_{h} m_{h} \varphi\left(Y_{h}^{*}\right) / \sum_{h} m_{h} \geq \sum_{h} \tilde{m}_{h} \varphi\left(\tilde{Y}_{h}^{*}\right) / \sum_{h} \tilde{m}_{h} \quad \text { for all } \varphi \in \Phi,
\end{gathered}
$$

which, using Lemma 1 again, may be expressed more succinctly as $\left(\mathbf{Y}^{*} \mid \mathbf{m}\right) \geq_{\mathrm{GL}}\left(\tilde{\mathbf{Y}}^{*} \mid \tilde{\mathbf{m}}\right)$. A heterogeneous sample of households is therefore unambiguously preferred in welfare terms if and only if the corresponding set of household equivalent income values weighted by their family size is preferred by the generalised Lorenz criterion. ${ }^{19}$

While this conclusion should not come as a surprise, it does answer one question which has remained unresolved for many years: whether, when undertaking welfare comparisons with

\footnotetext{
19 Strictly speaking, this has been demonstrated only when the number of households in the sample is fixed, along with the total number of individuals contained in those households. However these constraints may be omitted by appealing to the standard replication invariance condition.
} 
household data, the income variable should be aggregate household income, per-capita household income, or some other income concept; and whether households should be weighted equally, weighted according to family size, or weighted in some other fashion. ${ }^{20}$ The analysis here suggests that only one answer can be given to these questions: the appropriate income concept is the equivalent income value, and the appropriate weight is household size. Many empirical studies in the past have violated one or both of these requirements.

For heterogeneous inequality comparisons founded on equity preference, the unambiguous (nominal income) inequality criterion proposed in (62) may be reformulated for household data in a similar way to obtain $(\mathbf{y} ; \boldsymbol{\theta}) \geq_{\mathrm{I}}(\tilde{\mathbf{y}} ; \tilde{\boldsymbol{\theta}})$ if and only if $\left(\mathbf{Y}^{*} \mid \mathbf{A}\right) \geq_{\mathrm{L}}\left(\tilde{\mathbf{Y}}^{*} \mid \tilde{\mathbf{A}}\right)$, where $\mathbf{A}$ denotes the vector of household equivalence scale factors defined in (64). Here again the choice of income concept and weighting system are determined uniquely. But in this case households are weighted by their equivalence scale factor rather than family size. If, instead, heterogeneous inequality comparisons refer to inequality of living standards, then $(\mathbf{y} ; \boldsymbol{\theta})$ is no worse than $(\tilde{\mathbf{y}} ; \tilde{\boldsymbol{\theta}})$ if and only if $\left(\mathbf{Y}^{*} \mid \mathbf{m}\right) \geq_{\mathrm{L}}\left(\tilde{\mathbf{Y}}^{*} \mid \tilde{\mathbf{m}}\right)$, and the weighting system reverts to that based on family size.

\section{Summary and Conclusions}

This paper has tried to establish the principles that should govern the welfare and inequality analysis of heterogeneous populations when households are completely ordered by their level of material well-being or living standard. Two basic criteria suggested themselves: the equity preference requirement that welfare rises and inequality falls when income is transferred to those worse-off; and the compensation principle which demands that welfare and inequality are unchanged when a member of the population is replaced by another with the same standard of living. Both appear to be plausible and desirable properties but, as demonstrated in Proposition

\footnotetext{
${ }^{20}$ A detailed discussion is provided by Danziger and Taussig (1979) and Cowell (1984). See also Ringen (1991). These authors all favour using equivalent household incomes weighted by household size, as recommended here, although the reasons they give for doing so are essentially personal opinions of what constitutes reasonable empirical practice.
} 
1 , they are virtually incompatible. In order to proceed, therefore, one of the properties must be discarded.

The welfare analysis of Section 5, based on the compensation principle, was able to vindicate the traditional method of dealing with heterogeneous samples, which involves converting incomes into their equivalent income values and then treating those values as if they refer to a homogeneous population. It emerged, however, that conventional procedures rely on the assumption that equivalent incomes are obtained by deflating reported income values by a constant scale factor, independent of the level of income. Only then is it possible to choose freely the reference person (or household type) used for the equivalent income calculations. Although the equity preference property is abandoned, equity considerations are retained in the form of the PigouDalton condition for homogeneous samples, and may be expanded to respect the Weak Equity Axiom proposed by Sen (1973).

In contrast to welfare analysis, it seems more natural to base heterogeneous inequality comparisons on equity preference, as is done in Section 6. Here again it is difficult to progress unless equivalent incomes are derived using constant scale factors. Restricting attention to homothetic living standards enables the contributions of Glewwe (1991) and Ebert $(1995,1997$, 1999) to be assessed. But even with this restriction a variety of problems arise in attempting to characterise the 'unambiguous inequality ordering' for heterogeneous populations (i.e. the heterogeneous counterpart to Lorenz dominance), and in applying the results to situations likely to be encountered in practice. For these reasons it may be advisable to make a clear distinction between inequality of nominal incomes and inequality of living standards, and to focus on the latter when undertaking empirical studies. This approach justifies the traditional procedures used in the past, and offers a simple method of validating unambiguous inequality comparisons, via the Lorenz dominance ordering applied to distributions of equivalent incomes.

Finally, the paper considered the implications for heterogeneous samples of households distinguished by income and family size. Despite earlier suggestions that researchers are free to choose alternative concepts of income and to apply alternative system of weights to household 
observations, the analysis shows that this is not the case. Once the compensation principle is adopted for either welfare or inequality comparisons, the appropriate income concept is always the equivalent income value, and households must always be weighted according to the number of family members. 


\section{References}

Arnold, B.C. (1987): Majorization and the Lorenz Order: A Brief Introduction, Lecture Notes in Statistics, Vol. 43. Berlin: Springer-Verlag.

Atkinson, A.B. (1970): 'On the Measurement of Inequality', Journal of Economic Theory, 2, 244-263.

Atkinson, A.B. and F. Bourguignon, (1982): 'The Comparison of Multi-Dimensioned Distributions of Economic Status', Review of Economic Studies, 49, 183-201.

Atkinson, A.B., and F. Bourguignon (1987): 'Income Distributions and Differences in Needs' in G.R. Feiwel (ed.), Arrow and the Foundation of the Theory of Economic Policy. New York: Macmillan.

Blackorby, C. and D. Donaldson (1993): 'Adult Equivalence Scales and the Economic Implementation of Interpersonal Comparisons of Well-Being', Social Choice and Welfare $10,335-361$.

Bourguignon, F. (1989): 'Family Size and Social Utility: Income Distribution Dominance Criteria', Journal of Econometrics, 42, 67-80.

Buhmann, B., L. Rainwater, G. Schmaus and T. Smeeding (1988): 'Equivalence Scales, Wellbeing, Inequality and Poverty: Sensitivity Estimates Across Ten Countries using the Luxembourg Income Study (LIS) Database', Review of Income and Wealth, 34, 115-142.

Conniffe, D. (1992): 'The Non-constancy of Equivalence Scales', Review of Income and Wealth, $38,429-43$.

Coulter, F.A.E., F.A. Cowell and S.P. Jenkins (1992): 'Differences in Needs and Assessment of Income Distributions', Bulletin of Economic Research, 44, 77-124.

Cowell, F.A. (1984): 'The Structure of American Income Inequality', Review of Income and Wealth, 30, 351-375.

Danziger, S. and M.K. Taussig (1979): 'The Income Unit and the Anatomy of Income Distribution', Review of Income and Wealth, 25, 365-75.

Ebert, U. (1995): 'Income Inequality and Differences in Household Size', Mathematical Social Sciences, 30, 37-55.

Ebert, U. (1997): ‘Social Welfare When Needs Differ: An Axiomatic Approach', Economica, 
64, 233-44.

Ebert U. (1999): Using Equivalence Income of Equivalent Adults to Rank Income Distributions, Social Choice and Welfare 16, 233-258.

Ebert, U. and P. Moyes (2003): 'Equivalence Scales Reconsidered', Econometrica, 71, 319-43.

Fishburn, P.C. and R.G. Vickson (1978): 'Theoretical Foundations of Stochastic Dominance' in

G. A. Whitmore and M.C. Findlay (eds.) Stochastic Dominance. Lexington, Mass.: D. C. Heath and Co.

Fleurbaey, M., C. Hagneré and A. Trannoy (2003): 'Welfare Comparisons with Bounded Equivalence Scales', Journal of Economic Theory, 110, 309-336.

Foster, J.E., (1985): 'Inequality Measurement,' in P. E. Young, (ed.) Fair Allocation. Proceedings of Symposia in Applied Mathematics, Volume 33. Providence, R.I. : American Mathematical Society.

Glewwe, P. (1991): 'Household Equivalence Scales and the Measurement of Inequality: Transfers from the Poor to the Rich Could Decrease Inequality', Journal of Public Economics, 44, 211-216.

Haddad, L. and Kanbur, R. (1990): 'How Serious is the Neglect of Intra-household Inequality?', Economic Journal, 100, 866-881.

Jenkins, S.P. and P.J. Lambert (1993): 'Ranking Income Distributions When Needs Differ', Review of Income and Wealth, 39, 337-356.

Kolm, S.-C. (1969): 'The Optimal Production of Social Justice', in J. Margolis and H. Guitton (eds.), Public Economics. London: Macmillan.

Kolm, S.-C. (1977): ‘Multidimensional Egalitarianisms', Quarterly Journal of Economics, 91, $1-13$.

Lambert, P.J. and X. Ramos (2002): 'Welfare Comparisons: Sequential Procedures for Heterogeneous Populations', Economica, 69, 549-62.

Lazear, E.P. and R.T. Michael (1986): 'Estimating the Personal Distribution of Income with Adjustment for Within-family Variation', Journal of Labour Economics, 4, S216-S244.

Lazear, E.P. and R.T. Michael (1988): Allocation of Income Within the Household. Chicago: University of Chicago Press. 
Lewbel, A. (1989): 'Household Equivalence Scales and Welfare Comparisons', Journal of Public Economics, 39, 377-391.

Maasoumi, E. (1986): 'The Measurement and Decomposition of Multi-Dimensional Inequality', Econometrica, 54, 991-997.

Maasoumi, E. (1999): 'Multidimensioned Approaches to Welfare Analysis', in J. Silber (ed.) Handbook of income inequality measurement. Boston; Dordrecht and London: Kluwer Academic.

Moyes, P. and A.F. Shorrocks (1994): 'Transformations of Stochastic Orderings', in W. Eichhorn (ed.) Models and Measurement of Welfare and Inequality. Berlin: Springer Verlag.

Marshall, A.W., and I. Olkin (1979): Inequalities: Theory of Majorization and its Applications. New York: Academic Press.

Mukherjee, D. (2001): 'Measuring Multidimensional Deprivation', Mathematical Social Sciences, 42, 23-51.

Nelson, J.A. (1993): 'Household Equivalence Scales: Theory Versus Policy?', Journal of Labour Economics, 11, 471-493.

Nicol, C.J. (1994): 'Identifiability of Household Equivalence Scales through Exact Aggregation: Some Empirical Results', Canadian Journal of Economics, 27, 307-28.

Ok, E.A. and P.J. Lambert (1995): 'On Evaluating Social Welfare by Sequential Generalized Lorenz Dominance', Economics Letters 63:45-53.

Ooghe, E. (2003): 'Reference Type-independent Heterogeneous Social Evaluation', Economics Letters, 80, 227-231.

Pendakur, K. (1999): 'Semiparametric Estimates and Tests of Base-Independent Equivalence Scales', Journal of Economics, 88, 1-40.

Pollak, R.A. (1991): 'Welfare Comparisons and Situational Comparisons', Journal of Econometrics, 50, 31-48.

Pyatt, G. (1990): 'Social Evaluation Criteria' in C. Dagum and M. Zenga (eds.), Income and Wealth Distribution, Inequality and Poverty. Berlin: Springer-Verlag.

Ringen, S. (1991):'Households, Standard of Living, and Inequality', Review of Income and 
Wealth, 37, 1-13.

Roberts, K.W.S. (1980): 'Interpersonal Comparability and Social Choice Theory', Review of Economic Studies, 47, 421-439.

Sen, A.K. (1973): On Economic Inequality. Oxford: Clarendon Press.

Sen, A.K. (1997): On Economic Inequality. Expanded Edition with a substantial annexe. Oxford: Clarendon Press.

Sen, A.K. (1992): Inequality Reexamined. Oxford: Clarendon Press.

Shorrocks, A.F. (1983): 'Ranking Income Distributions’, Economica, 50, 3-17.

Thomas, D. (1990): 'Intra-household Resource Allocation: An Inferential Approach', Journal of Human Resources, 25, 635-664.

Trannoy, A. (2003): 'About the Right Weights of the Social Welfare Function when Needs Differ', Economics Letters, 81, 383-387.

Tsui, K.Y. (1995): 'Multidimensional Generalizations of the Relative and Absolute Inequality Indices: The Atkinson-Kolm-Sen Approach', Journal of Economic Theory, 67, 251-65.

Tsui, K.Y. (1999): 'Multidimensional Inequality and Multidimensional Generalized Entropy Measures: An Axiomatic Derivation', Social Choice and Welfare, 16, 145-57.

Tsui, K.Y. (2002): 'Multidimensional Poverty Indices', Social Choice and Welfare, 19, 69-93.

Whiteford, P. (1985): A Family's Needs: Equivalence Scales, Poverty and Social Security. Canberra: Government Printer. 
Figure 1: Social Indifference Curves for Heterogeneous Income Distributions

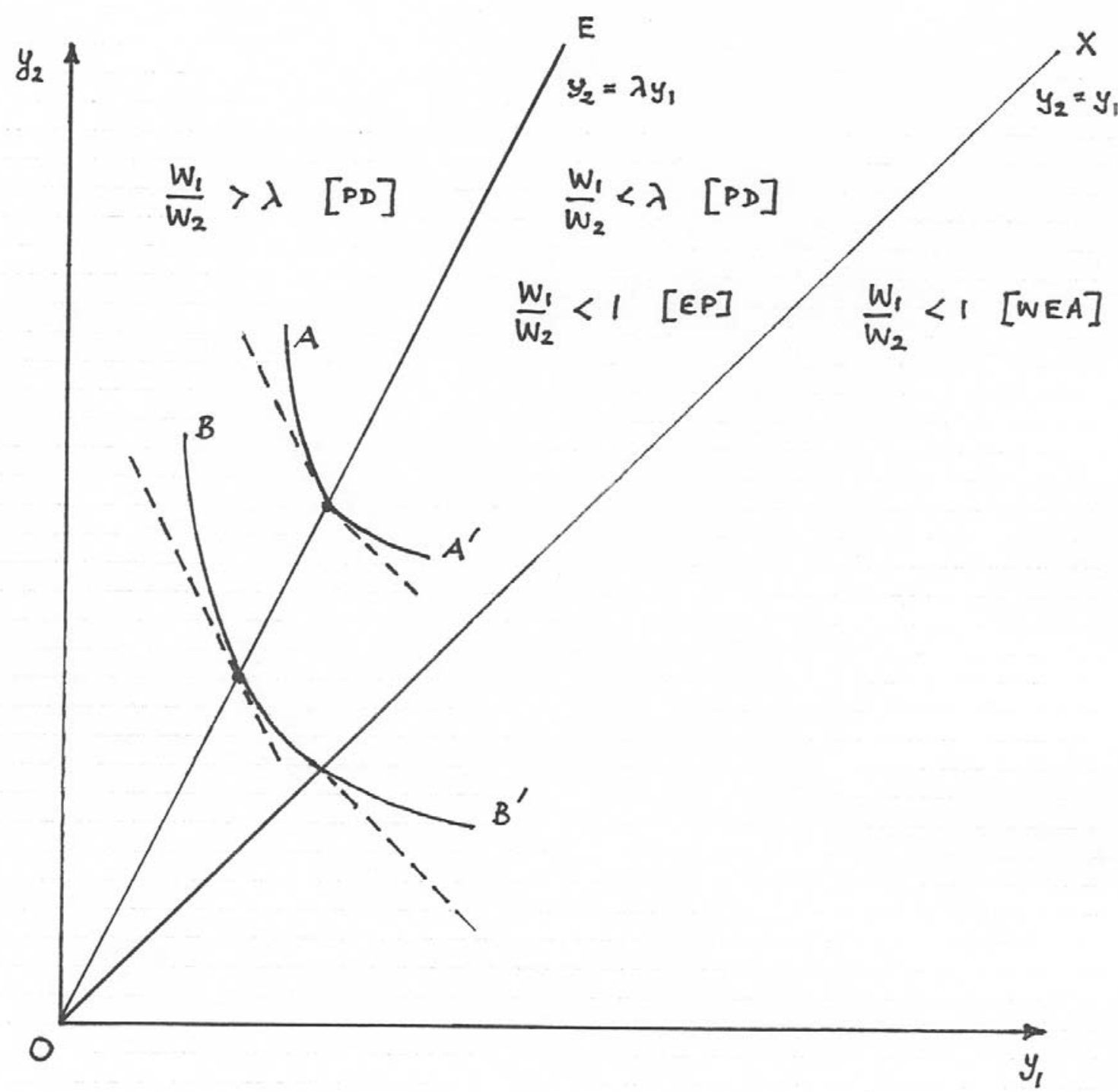

Note: Restrictions on the marginal social rate of substitution result from equity preference [EP], the Pigou-Dalton condition [PD], and the Weak Equity Axiom [WEA]. 